\title{
Effectiveness of Mindfulness-based Cognitive Therapy on Cognitive-Behavioral Avoidance and Mental Rumination in Comorbidity of Social Anxiety and Depression Patients
}

\section{ARTICLE INFO}

\section{Article Type}

Original Research

\section{Authors}

Ghadampour E. ${ }^{1} P h D$,

Radmehr P.* MSc,

Yousefvand L. ${ }^{2} M S C$

How to cite this article
Ghadampour E, Radmehr P, Yo-
usefvand L. Effectiveness of Mi-
ndfulness-based Cognitive The-
rapy on Cognitive-Behavioral A-
voidance and Mental Ruminati-
on in Comorbidity of Social Anx-
iety and Depression Patients. Ho-
rizon of Medical Sciences. 2017;
$23(2): 141-148$.

*Psychology Department, Literature \& Humanities Faculty, Lorestan University, Lorestan, Iran

${ }^{1}$ Psychology Department, Literature \& Humanities Faculty, Lorestan University, Lorestan, Iran

${ }^{2}$ Educational Management Department, Literature \& Humanities Faculty, Lorestan University, Lorestan, Iran

\section{Correspondence}

Address: Khorramabad, Kamalvand, Kiloumetr5 KhorramabadTehran, Lorestan University, Literature \& Humanities Faculty, Psychology Department Phone: +98 (83) 48224904 Fax: -

radmehr.p12@gmail.com

\section{Article History}

Received: May 20, 2016

Accepted: November 8, 2016

ePublished: March 25, 2017

\section{A B S T R A C T}

Aims As one of the most prevalent disorders in the adolescents, the comorbidity of social anxiety disorder and depression leads to bad outcomes for them. The aim of the study was to determine the effectiveness of the midfulness-based cognitive therapy on the cognitivebehavioral avoidance and mental rumination in patients with comorbidity of social anxiety and depression.

Materials \& Methods In the controlled follow-up pretest-posttest quasi-experimental study, 30 female high-school students with the social anxiety and depression comorbiduty were studied in Khorramabad in the academic year 2015-16. The subjects, selected via purposeful sampling method, were randomly divided into two 15-person groups including experimental and control groups. Data was collected by the structured clinical interview for Axis 1 disorders, the social anxiety questionnaire for the adolescents, Beck depression inventorysecond edition, the cognitive-behavioral avoidance scale, and the ruminative responces scale. Eight 2-hour group mindfulness-based cognitive-therapy training sessions (one session per week) were conducted in experimental group, while control group received no intervention. Finally, posttest was conducted in both groups and a follow-up step was conducted 2 month latter. Data was analyzed by SPSS 19 software using multi-variable covariance analysis test. Findings The mean scores of the cognitive-behavioral avoidance and mental rumination items in the posttest and follow-up steps significantly decreased in experimental group compared to control group $(\mathrm{p}<0.01)$.

Conclusion The mindfulness-based cognitive therapy reduces the cognitive-behavioral avoidance, as well as the mental rumination, in the patients with the social anxiety and depression comorbidity.

Keywords Mindfulness Cognitive Therapy; Cognitive-Behavioral Avoidance; Social Anxiety Disorder; Depression

\section{CIT A T I O N L INKS}

[1] Social anxiety and ... [2] A meta-analytic review ... [3] Relationship between social phobia ... [4] Clinical experiences in conducting ... [5] Interpretation bias and social [6] Social performances deficits in social ... [7] Psychometric properties of the social [8] Social anxiety disorder/social phobia ... [9] Decrease in self-esteem mediates [10] Lifetime prevalence and age ... [11] A comparative study about the intensity ... [12] Social anxiety disorder ... [13] Comorbid depression and anxiety ... [14] Associations between Peer victimization, self-reported ... [15] Comorbidity of anxiety and unipolar ... [16] Responses to depression and their effects ... [17] The relationship between cognitive ... [18] The treatment of social anxiety ... [19] Avoidance and depression ... [20] Experiential avoidance in idiographic ... [21] An evaluation of an integrated ... [22] Impact of rumination ... [23] Testing the cognitive catalyst ... [24] Rumination, worry ... [25] The effects of rumination ... [26] Rumination as a transdiagnostic ... [27] Worry and rumination ... [28] The response styles theory ... [29] Cognitive factors that ... [30] A roadmap to rumination ... [31] The role of rumination ... [32] Comorbid social anxiety disorder ... [33] Impactofanxiety disorder ... [34] Depression and generali ... [35] Exploring the effectiveness... [36] Cognitive behavioral group ... [37] Attention training in individuals ... [38] The effect of mindfulness ... [39] Mindfulness-based therapy ... [40] Efficacy of the third wave of behavioral therapies ... [41] Adapting mindfulness ... [42] The benefits of being ... [43] Cognitive therapy ... [44] Mindfulness-based stress ... [45] The effectivness of combined ... [46] Web-based Mindfulness ... [47] The etiology of social phobia ... [48] Kaplan \& Sadock's Comprehensive ... [49] Group therapy ... [50] The effectiveness of ... [51] Reliability and feasibility ... [52] Examine the psychometric ... [53] The relationship ... [54] Rumination and cognitive ... [55] Prevention of relapse ... [56] Efficacy of mindfulness ... [57] The effects of ... 
دادهاند كه اين اختلال در نوجوانان با مسايل و مشكلات بى شمارى إى إي

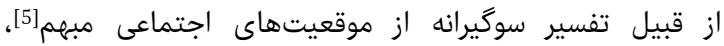

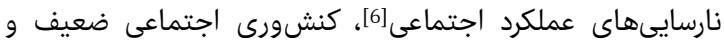
ادراكهاى منفى [7] رابطه دارد. اين اختلال همبودى بالايى با ساير اختلالات اضطرابى خلقاد القى داردا

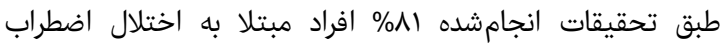

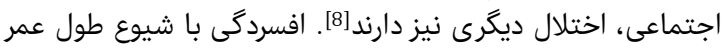

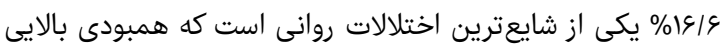

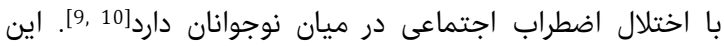

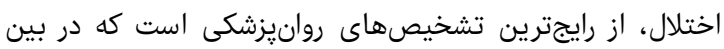

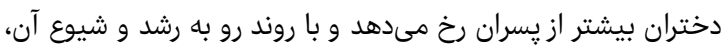

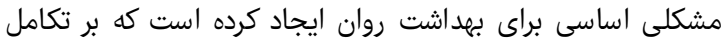

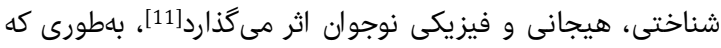

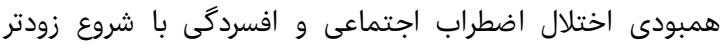

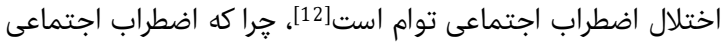

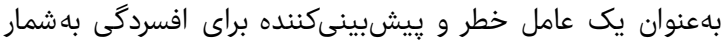

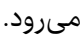
مبتلايان به اختلال اضطراب اجتماعى اكثراً از نظر بالينى افسرده إنها

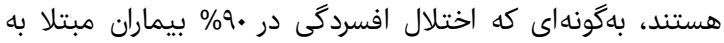

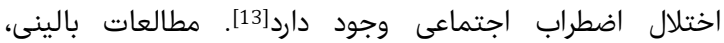

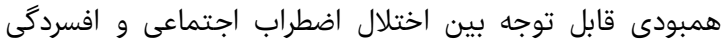

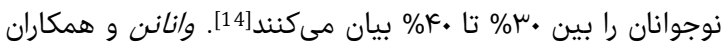

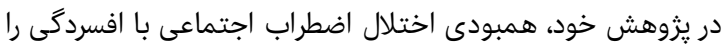

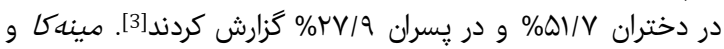

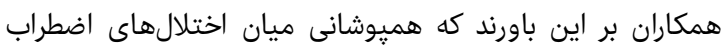

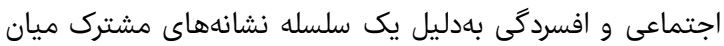

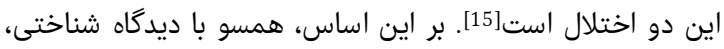

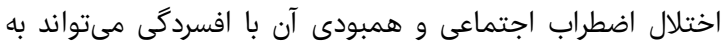

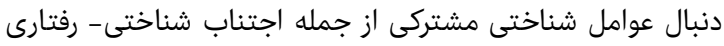

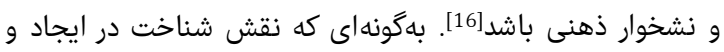

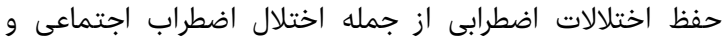

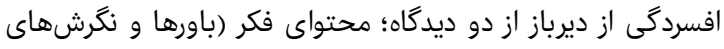

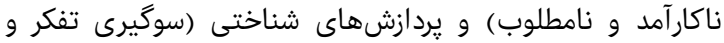

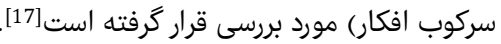

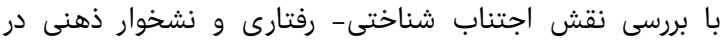

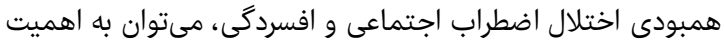

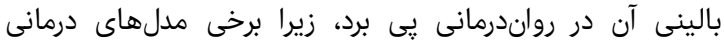

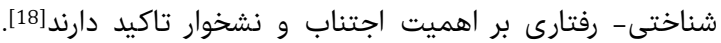

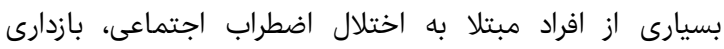

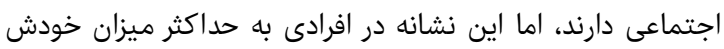

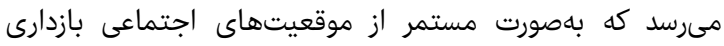

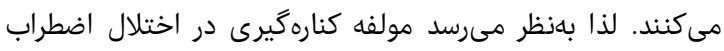

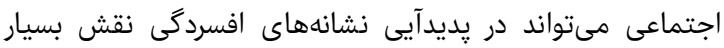

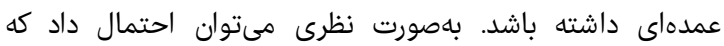

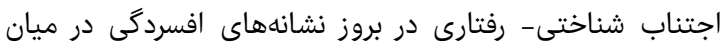

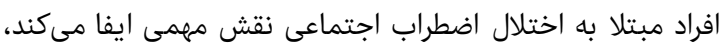

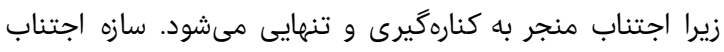

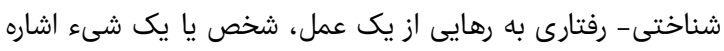

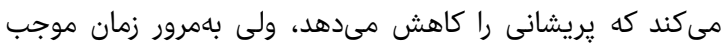

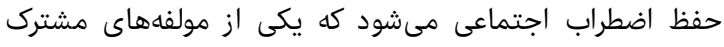

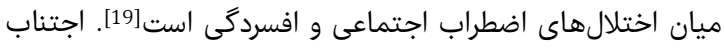

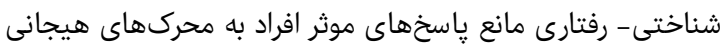
دوره سץ، شماره r، بهار
اثربخشى درمان شناختى مبتنى بر ذهنآكاهى بر

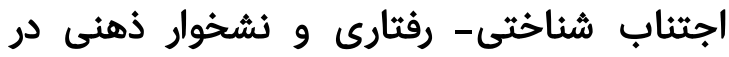

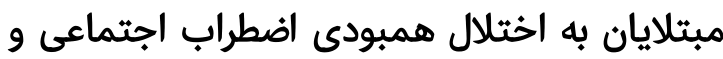
افسردگى مبتلان

عزت عزاله قدميور PhD كروه روانشناسى، دانشكده ادبيات و علوم انسانى، دانشكاه لرستان، لرستان، ايران

MSc "يروانه رادمهر كروه روانشناسى، دانشكده ادبيات و علوم انسانى، دانشكاه لرستان، لرستان، ايران

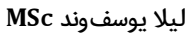
كروه مديريت آموزشى، دانشكده ادبيات و علوم انسان انسانى، دانشكاه لرستان،

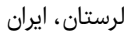

جكيده

اهداف: همبودى اختلال اضطراب اجتماعى و افسردگى از شايعترين مشكلات

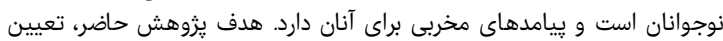

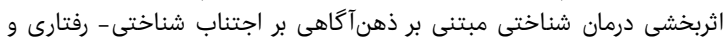

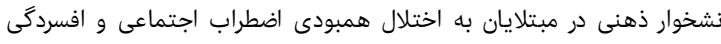

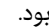

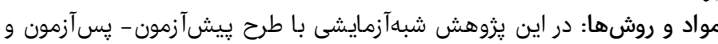

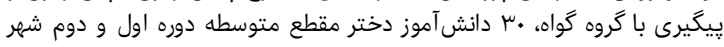

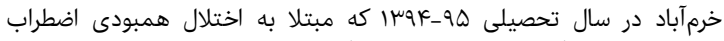

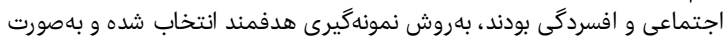

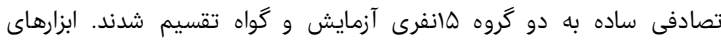

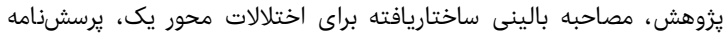

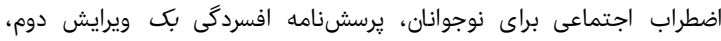

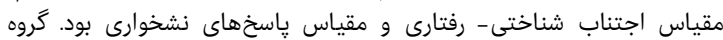

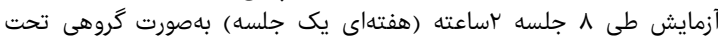

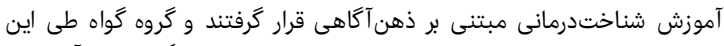

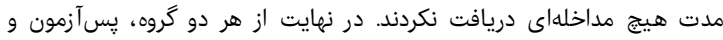

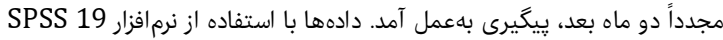

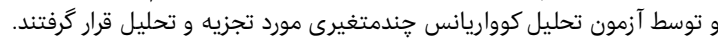

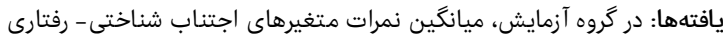

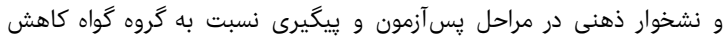

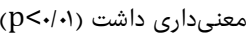

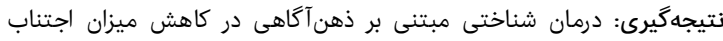

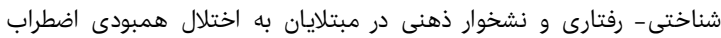
اجتماعى و افسردگى موثر است. كليدوازمها: شناخت درمانى مبتنى بر ذهن آكاهى آهى، اجتناب شناختى- رفتارى، اضطراب

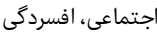

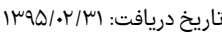

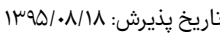
radmehr.p12@gmail.com :نويسنده مسئول:

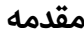

اختلال اضطراب اجتماعى يك حالت هيجانى است كه با نكرانى و

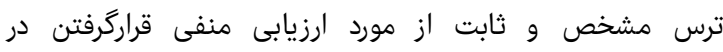

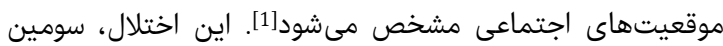

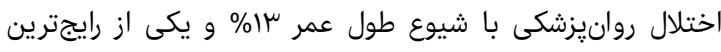

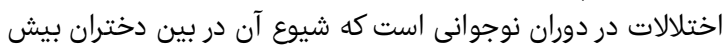

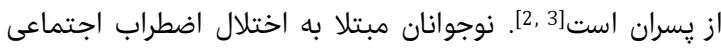

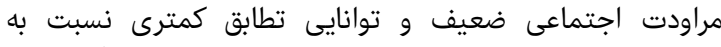

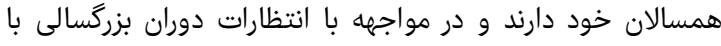

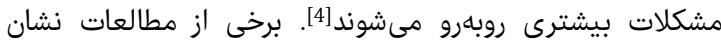

فصل نامه علمى - يزروهشى افق دانش 


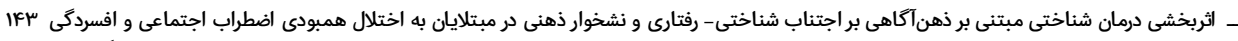

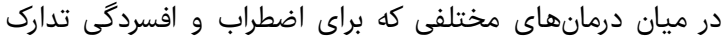

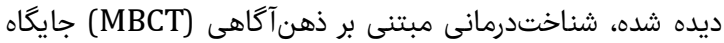

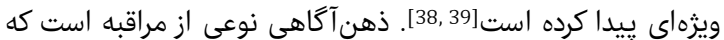

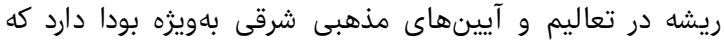

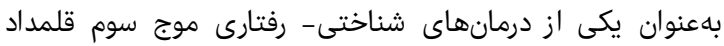

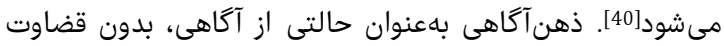

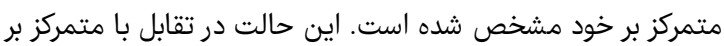

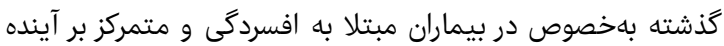

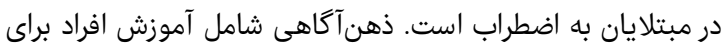

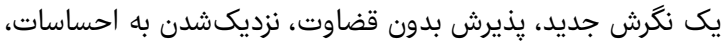

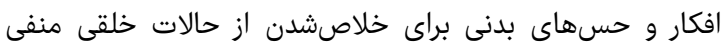

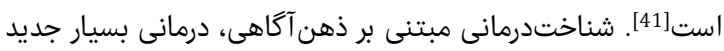

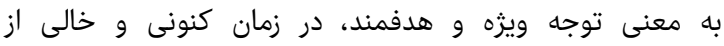

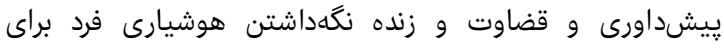

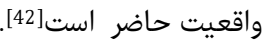

اسميتس و همكاران در يزوهش خود به إيت اين نتيجه رسيدند كه

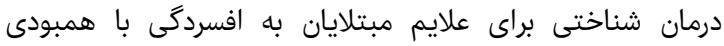

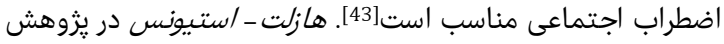

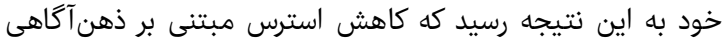

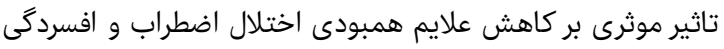

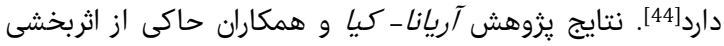

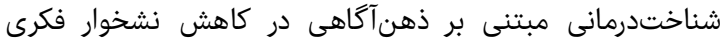

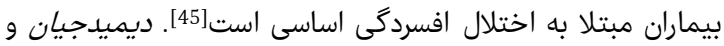

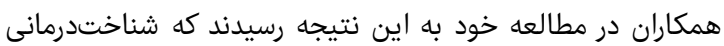

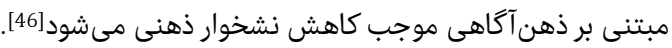

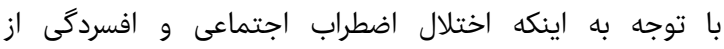

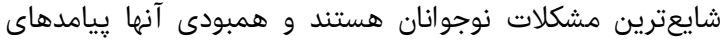

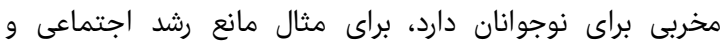

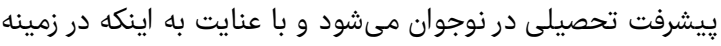

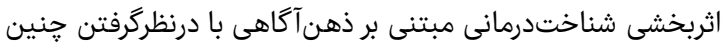

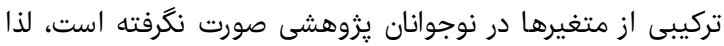

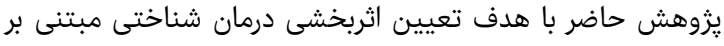

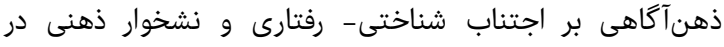

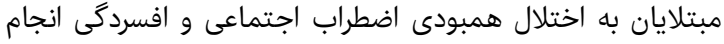

شد.

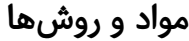

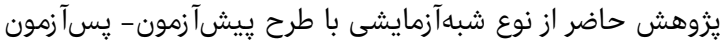

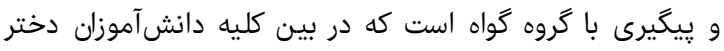

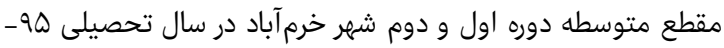

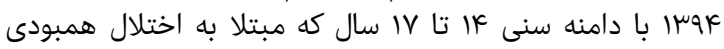

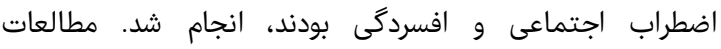

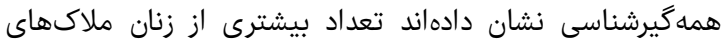

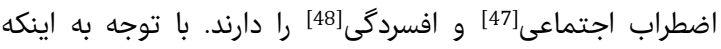

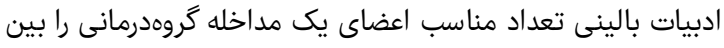

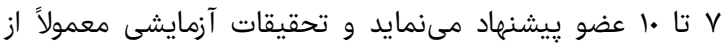

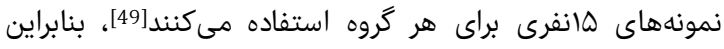

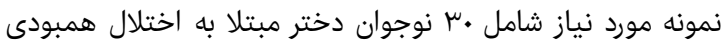

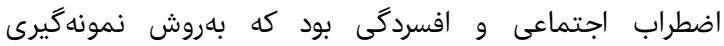

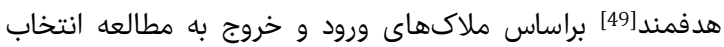

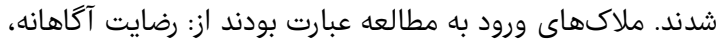

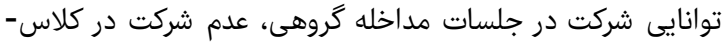

و جايكزينى راهبردهاى مديريت هيجان مى شود و به همين دين دليل

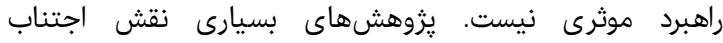

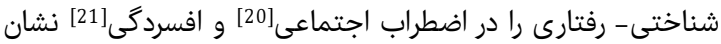

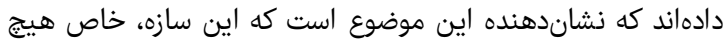

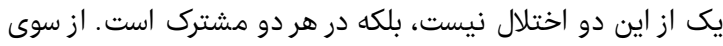

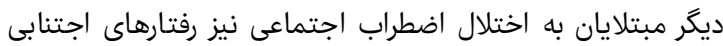

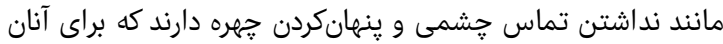

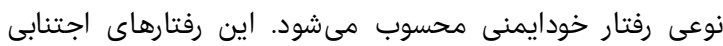

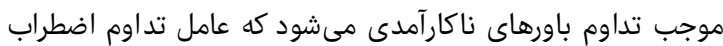

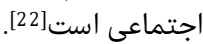

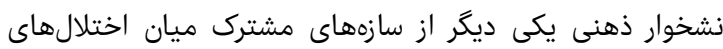

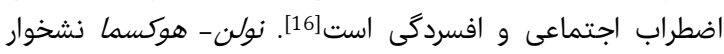

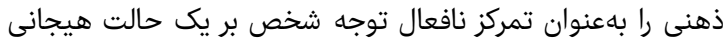

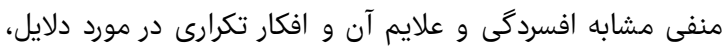

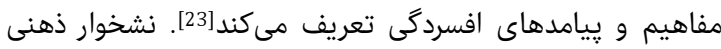

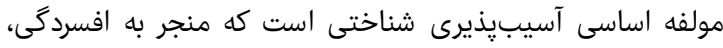

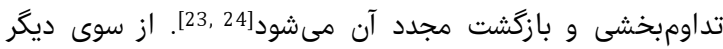

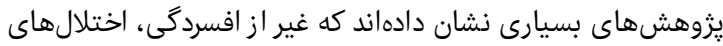

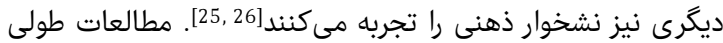

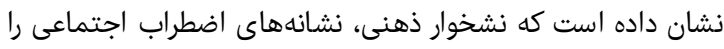

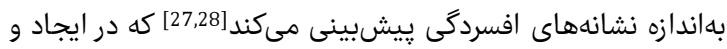

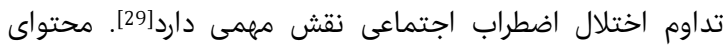

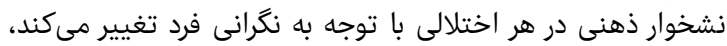

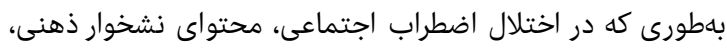

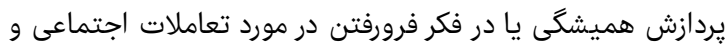

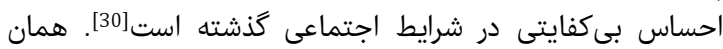

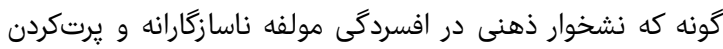

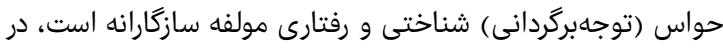

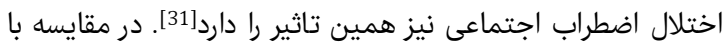

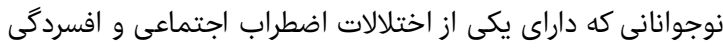

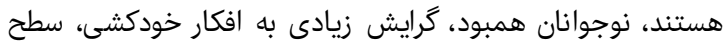

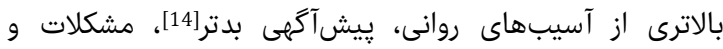

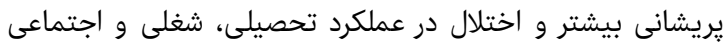

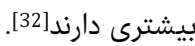

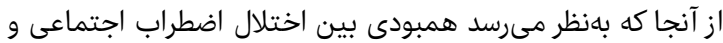

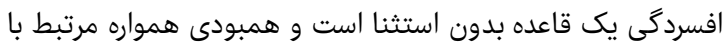

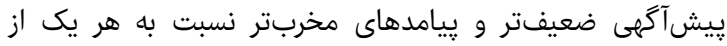

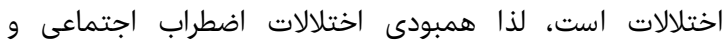

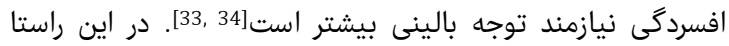

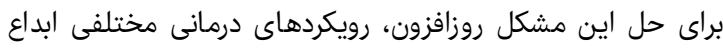

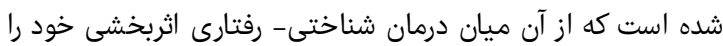

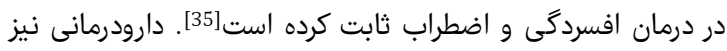

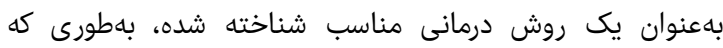

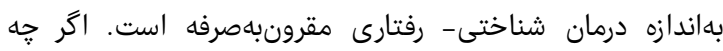

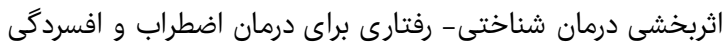

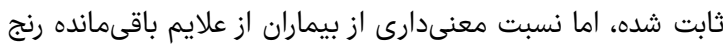

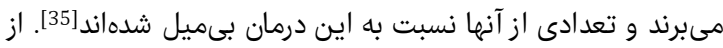

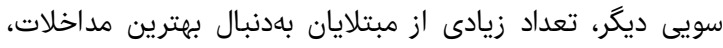

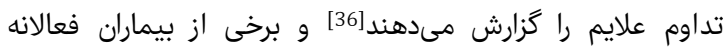

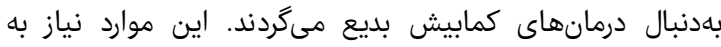

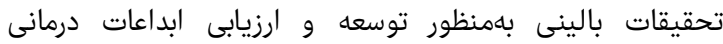

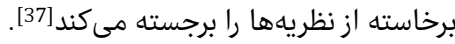

Volume 23, Issue 2, Spring 2017 


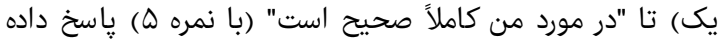

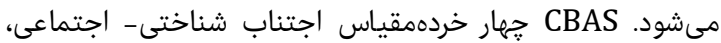

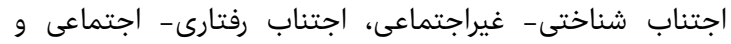

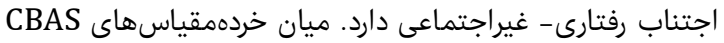

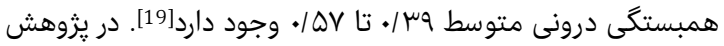

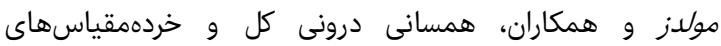

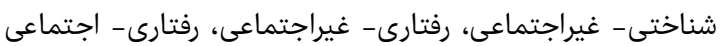

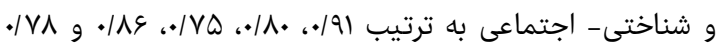

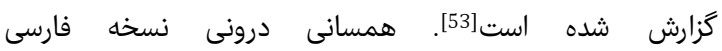

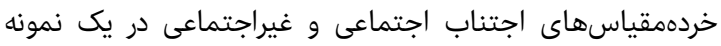

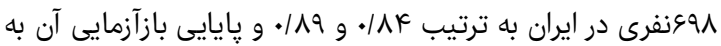

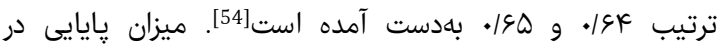

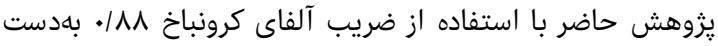

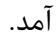

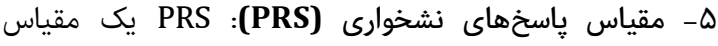

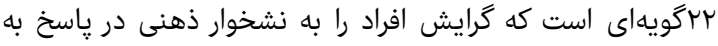

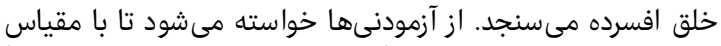

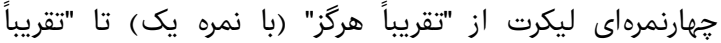

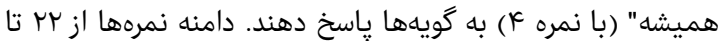

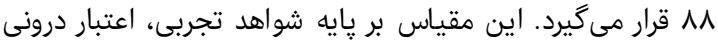

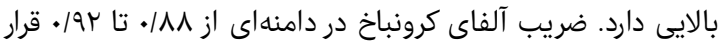

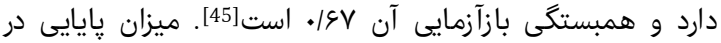

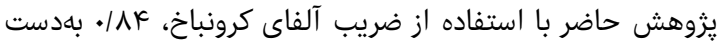

جدول () ساختار كلى جلسات آموزشى شناختدرمانى مبتنى بر ذهن آكاهى جلسه اول

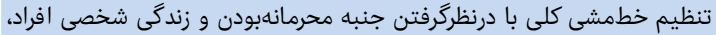

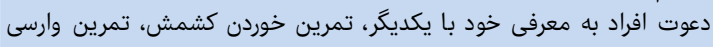

جلسه دوم توليف

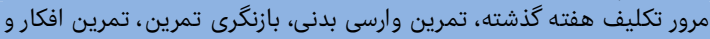

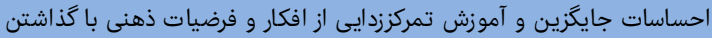

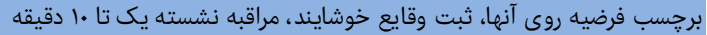

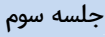

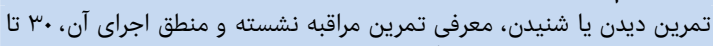

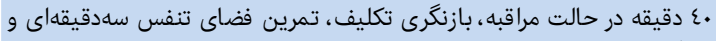

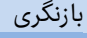
جلسه جهارم

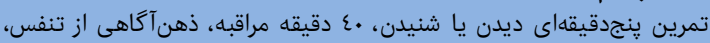

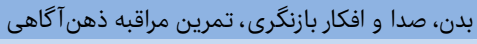
جلسه ينجم

آموزش اينكه قبل از هر تغييرى بايد خود را به همان گَونهاى كه هستيم احسيم

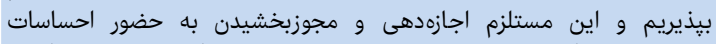

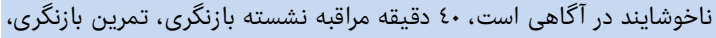

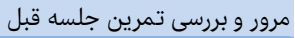

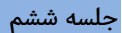

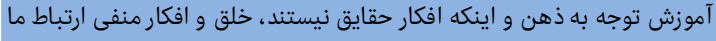

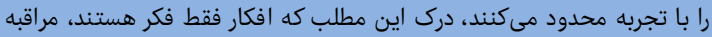

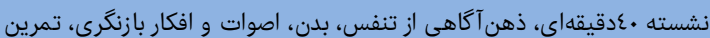

جلسه هفتم

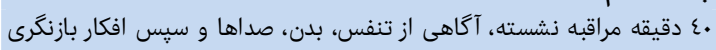

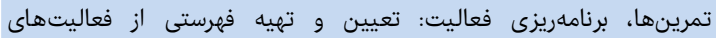

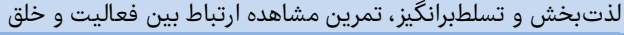
جلسه هشتم بازنكرى مطالب گذشته و جمعبندى

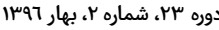

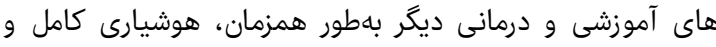

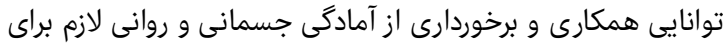

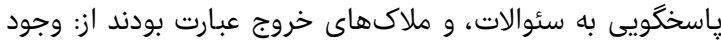

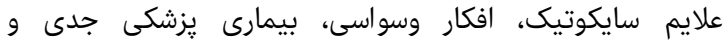

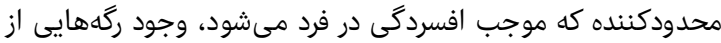

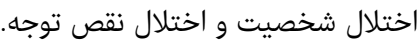

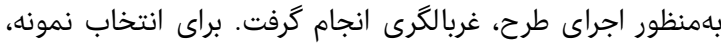

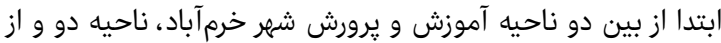

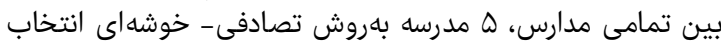

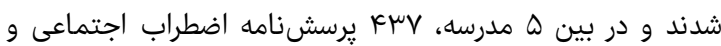

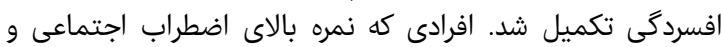

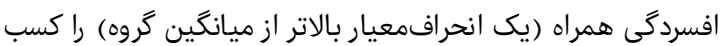

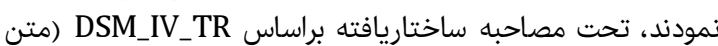

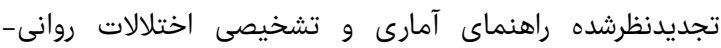

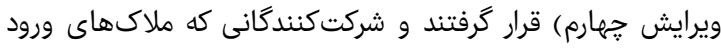

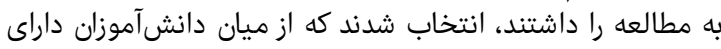

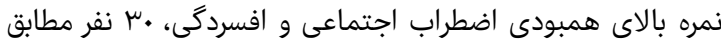

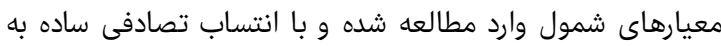

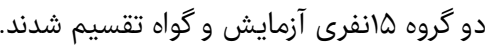

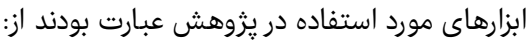

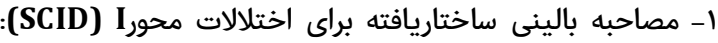

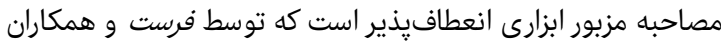

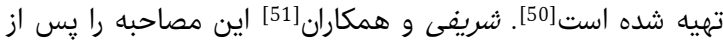

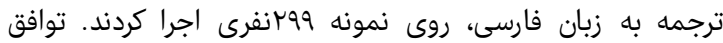

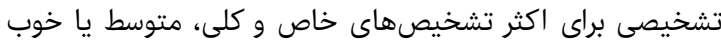

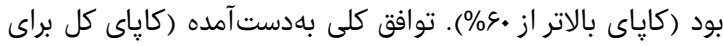

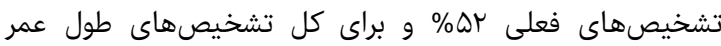

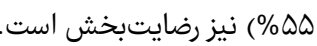

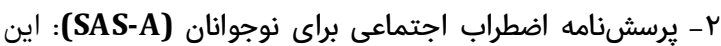

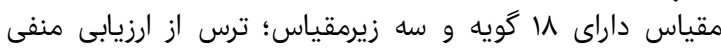

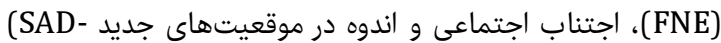

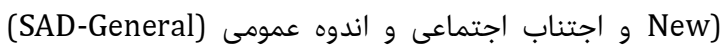

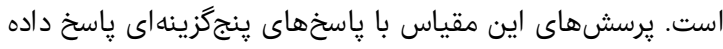

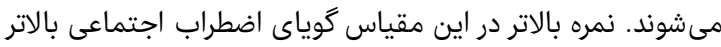

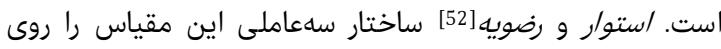

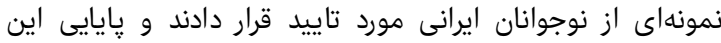

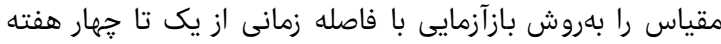

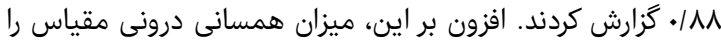

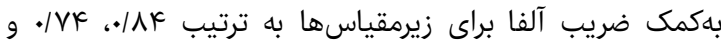

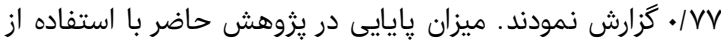

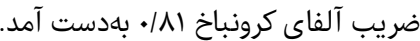

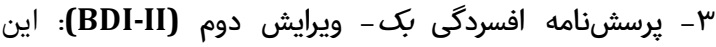

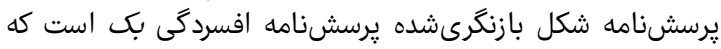

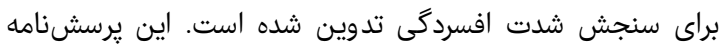

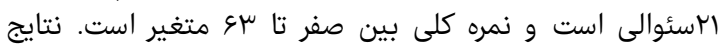

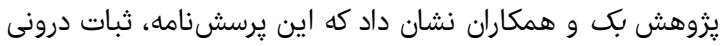

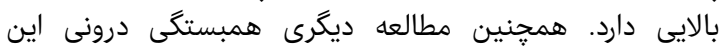

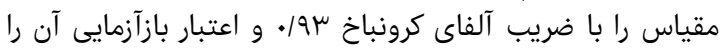

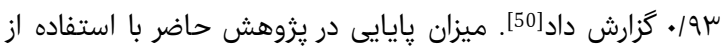

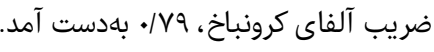

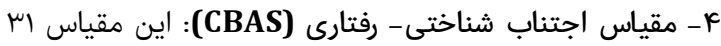

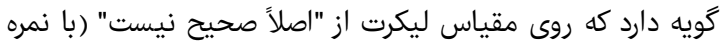
فصل نامه علمى - يُوهشى افق دانش 


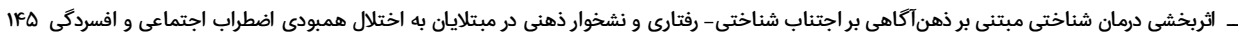

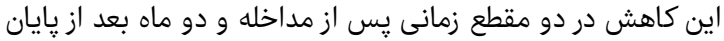

مداخله قابل مشاهده بود.

با مرور ييشينه يزوهش، تحقيقى كه به اثربخشى درمان شناختى

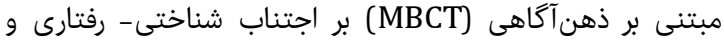

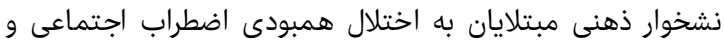

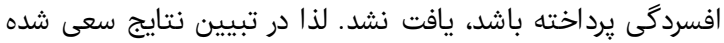

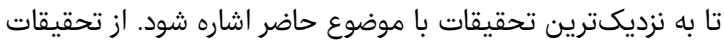

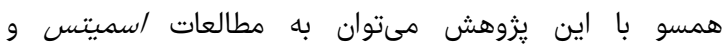

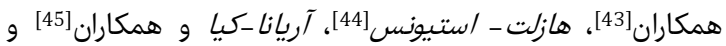

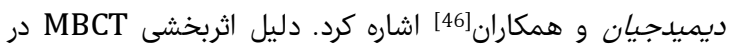

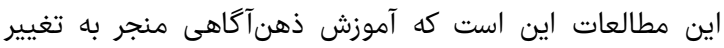

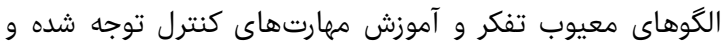

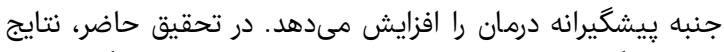

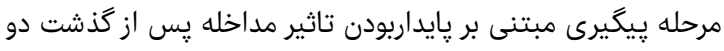

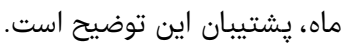

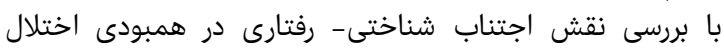

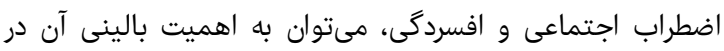

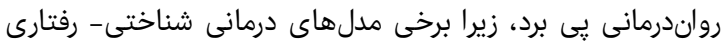

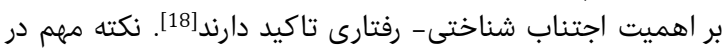

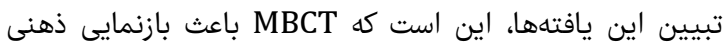

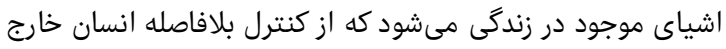

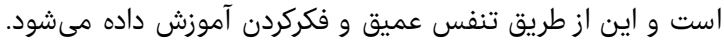

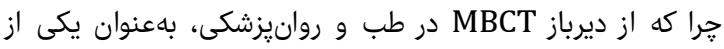

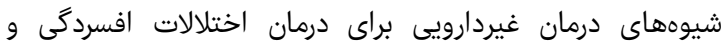

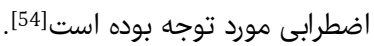

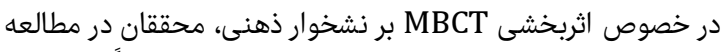

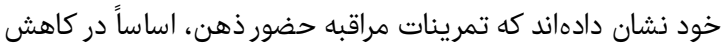

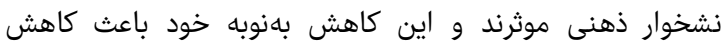

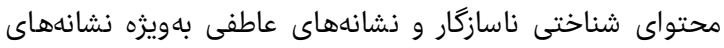

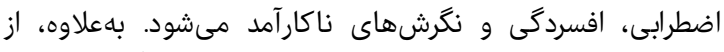

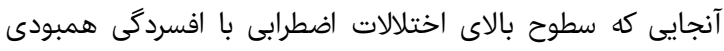

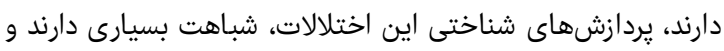

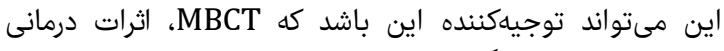

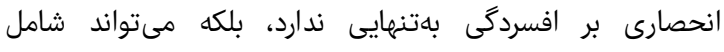
اختلالات اضطرابى نيز شود[55].

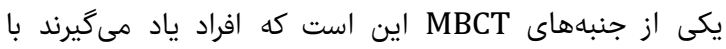

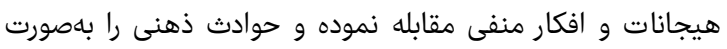

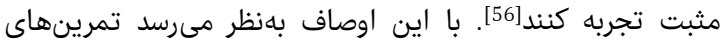

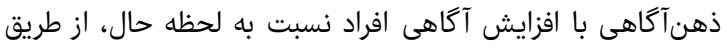

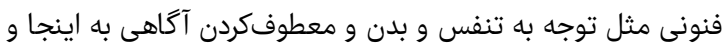

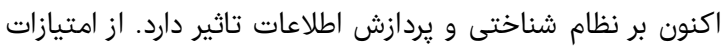

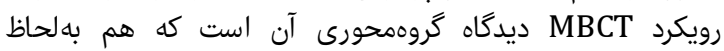

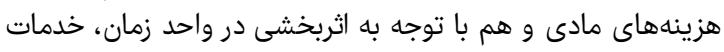

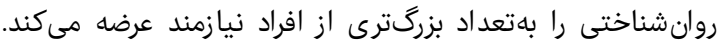

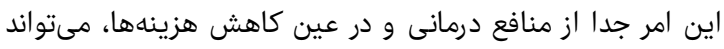

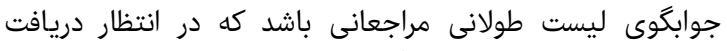

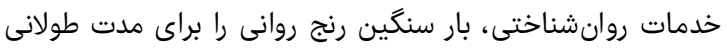

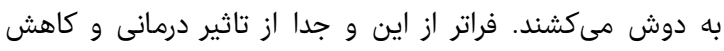

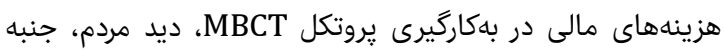

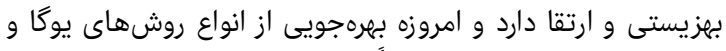

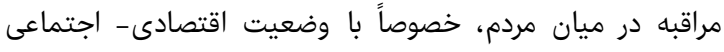

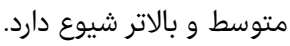

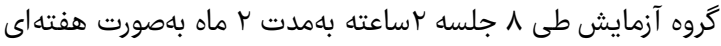

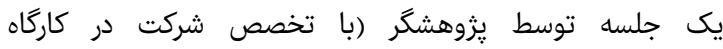

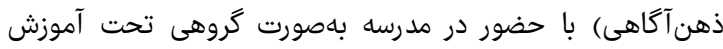

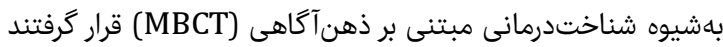

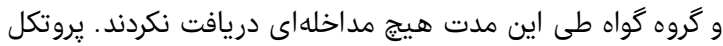

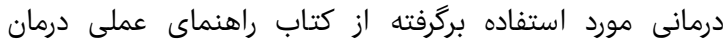
شناختى مبتنى بر حضور ذهن (نوشته سكال و و همكاران، سال

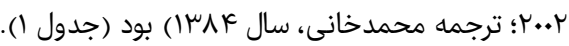

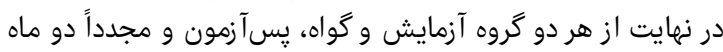

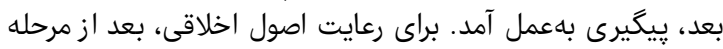

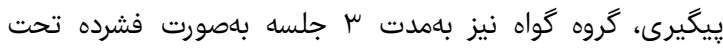

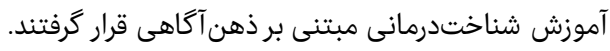

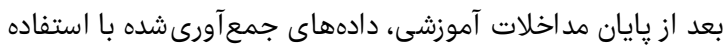

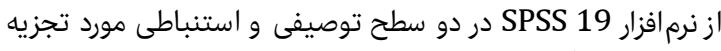

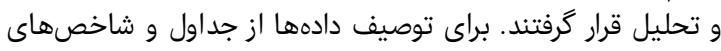

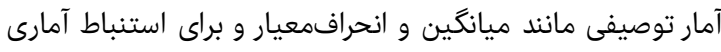

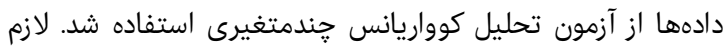

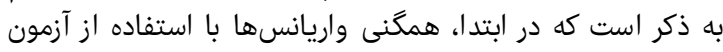

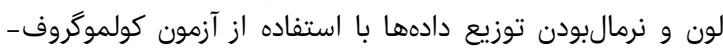
اسميرنوف مورد بررسى قرار گرفت.

يافتهها

ميانگين سنى در گروه آزمايش

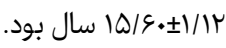

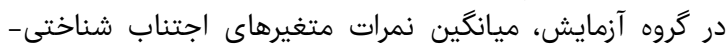

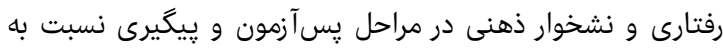

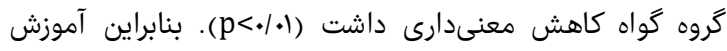

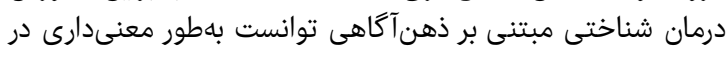

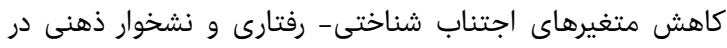

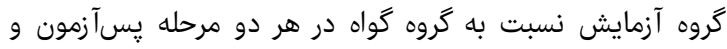

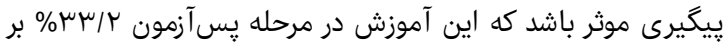

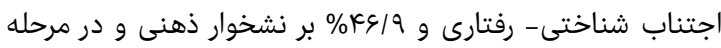

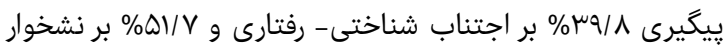

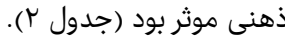

جدول r) ميانكَين آمارى نمرات اجتناب شناختى - رفتارى و نشخوار ذهنى در

\begin{tabular}{|c|c|c|}
\hline كروه گواه & گروه آزمايش & متغيرها \\
\hline & & اجتناب شناختى - رفتارى \\
\hline$\wedge \varepsilon / \vee \Delta \pm F / \Delta \Lambda$ & $\Lambda V / \Delta \Delta \pm F / \cdot 1$ & 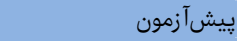 \\
\hline$\Lambda F / \gamma \wedge \pm F / \vee F$ & $V N / / H_{ \pm} \pm F / F \Delta$ & يس"آزمون \\
\hline$\wedge \ll / \cdot \perp \pm \Delta / \Delta \Delta$ & $V \varepsilon / 9 \Delta \pm F / .9$ & ي ييكيرى \\
\hline & & ن شخوار ذهنى \\
\hline$\Delta 1 / 1 \xi \pm \mu / K q$ & $\Delta \cdot / १ \Delta \pm \varepsilon / k \mu$ & 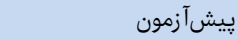 \\
\hline $0 \cdot 1 \cdot \mu_{ \pm}+\mu / \cdot r$ & $\kappa \mu / K \lambda \pm \varepsilon / V \mu$ & يسآزمون \\
\hline$\kappa q / \psi \wedge \pm \Psi / \mu v$ & $\kappa \mid / \wedge r \pm \Delta / \cdot \mu$ & يِيخيرى \\
\hline
\end{tabular}

هدف يزوهش حاضر، تعيين اثربخشى درمان شناختى مبتنى بر

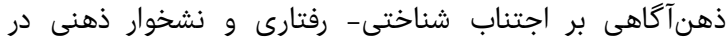

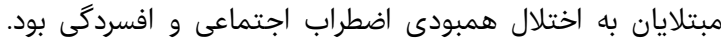
نتايج نشان داد كه جلسات هشت اختانه

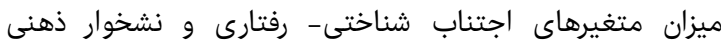

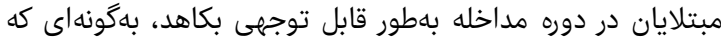


منابع

1- Kingsbury M, Coplan R. Social anxiety and interpretation of ambiguous text messages. Comput Hum Behav. 2015;54(C):368-79.

2- Teubert D, Pinquart M. A meta-analytic review on the presentation of symptom of anxiety in children adolescents. J Anxiety Disord. 2011;25(8):1046-59.

3- Väänänen JM, Fröjd $S$, Ranta $K$, Marttunen $M$, Helminen M, Kaltiala-Heino R. Relationship between social phobia and depression differs between boys and girls in mid-adolescen. J Affect Disord. 2011;133(12):97-104.

4- McAleavey AA, Castonguay LG, Goldfried MR. Clinical experiences in conducting cognitive behavioral therapy for social phobia. Behav Ther. 2014;45(1):21-35.

5- Miers AC, Blote AW, Bogels SM, Westenberg PM. Interpretation bias and social anxiety in adolescents. J Anxiety Disord. 2008;22(8):1462-71.

6- Voncken MJ, Bogels SM. Social performances deficits in social anxiety disorder: Reality during conversation and biased perception during a speech. J Anxiety Disord. 2008;22(8):1384-92.

7- Puklek M, Videc M. Psychometric properties of the social anxiety scale for adolescents (SAS-A) and its relation to positive imaginary audience and academic performance in Slovene adolescents. Stud Psychol. 2008;50(1):49-65.

8- den Boer JA. Social anxiety disorder/social phobia: Epidemiology, diagnosis, neurobiology and treatment. Compr Psychiatry. 2000;41(6):405-15.

9- Väänänen JM, Isomaa R, Kaltiala-Heino R, Fröjd $S$, Helminen M, Marttunen M. Decrease in self-esteem mediates the association between symptoms of social phobia and depression in middle adolescence prospective population cohort study. BMC Psychiatry. 2014;14:79.

10- Kessler RC, Berglund P, Demler O, Jin R, Merikangas KR, Walters EE. Lifetime prevalence and age-of-onset distributions of DSM-IV disorders in the National Comorbidity Survey Replication. Arch Gen Psychiatry. 2005;62(6):593-602.

11- Habibpour Z, Sharifi A. A comparative study about the intensity of depression among girls and boys in teenage period. J Urmia Nurs Midwifery Fac. 2009;7(1):10-2. [Persian]

12- Stein MB, Fuetsch M, Müller N, Höfler M, Lieb R, Wittchen HU. Social anxiety disorder and the risk of depression: A prospective community study of adolescents and young adults. Arch Gen Psychiatry. 2001;58(3):251-6.

13- Gorman JM. Comorbid depression and anxiety spectrum disorder. Depress Anxiety. 19961997;4(4):160-8.

14- Ranta K, Kaltiala-Heino R, Pelkonen M, Marttunen M. Associations between Peer victimization, self-reported depression and social phobia among adolescents: The role of comorbidity. J Adolesc. 2009;32(1):77-93

15- Mineka S, Watson D, Clark LA. Comorbidity of anxiety and unipolar mood disorder. Annu Rev Psychol. 1998;49:377-412.

16- Nolen-Hoeksema S. Responses to depression and their effects on the duration of depressive episodes. J Abnorm Psychol. 1991;100(4):569-82.

17- Fawzy TI, Hecker JE, Clark J. The relationship between cognitive avoidance and attentional bias forsnake-related thoughts. J Anxiety Disord. 2006;20(8):1103-7.

دوره سץr، شماره r، بهار דوسا
محدوديتهايى در مطالعه وجود داشت كه لازم است دارد در تفسير و

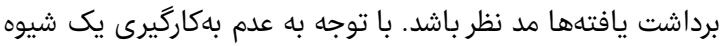

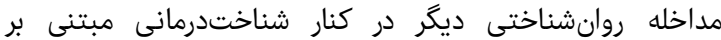

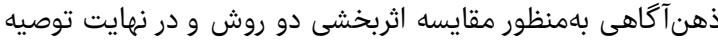

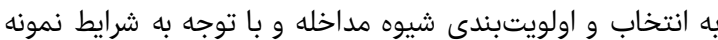

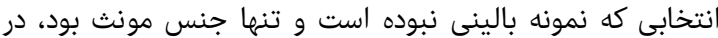

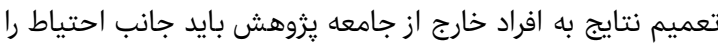

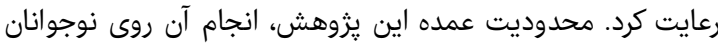

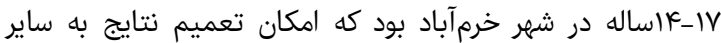

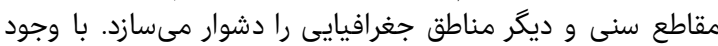

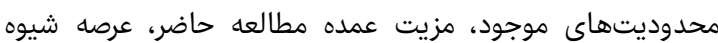

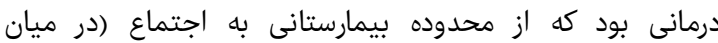

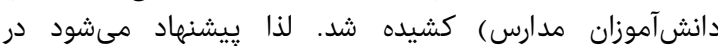

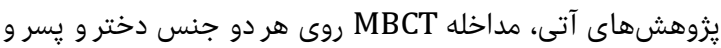

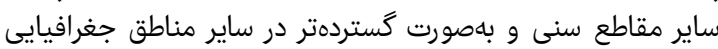

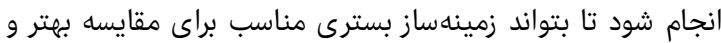

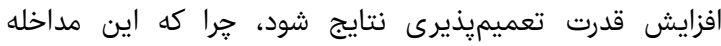

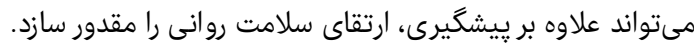

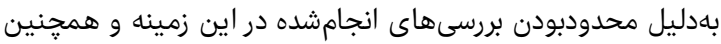

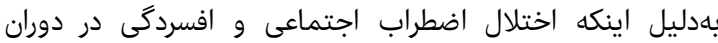

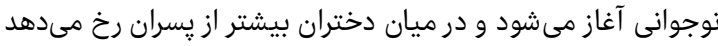

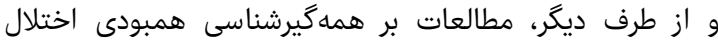

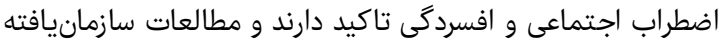

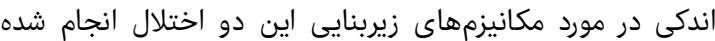

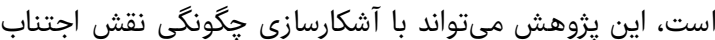

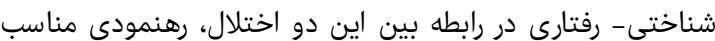

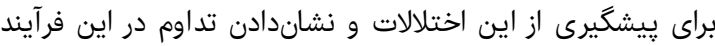

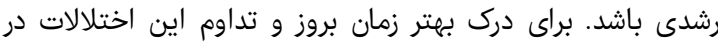

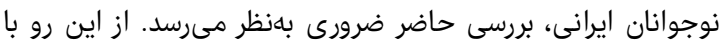

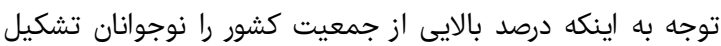

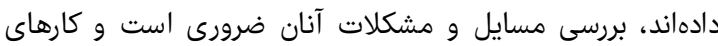
يثزوهشى بيشترى را مىطلبد.

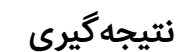

درمان شناختى مبتنى بر ذهن ذآكاهى در كاهش ميزان آدان اجتناب

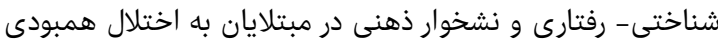

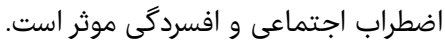

تشكر و قدردانى: در يايان از مساعدتهاى تمامى عوامل بهويزه

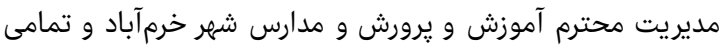

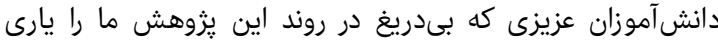

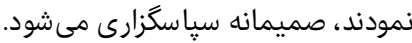

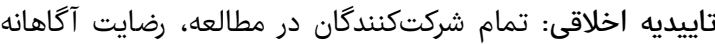

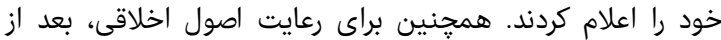

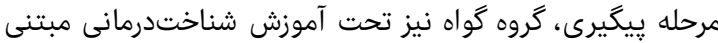
بر ذهن آكاهى قرار كرفتند.

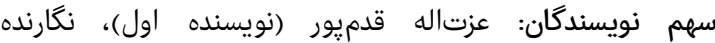

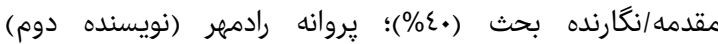

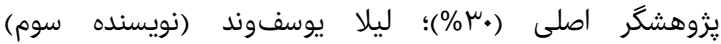

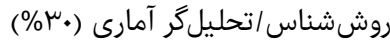

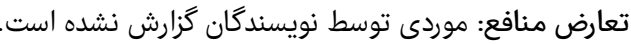
منابع مالى: اين مقاله تحت حمايت مالى مالى هيج سازمانى نبودئ نشان 


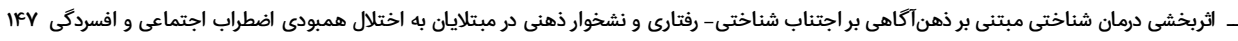

intervention across diverse populations. J Cogn Behav Pract. 2012;19(3):472-82.

36- McEvoy PM, Perini SJ. Cognitive behavioral group therapy for social phobia with or without attention training: A controlled trial. J Anxiety Disord. 2009;23(4):519-28.

37- Amir N, Beard C, Taylor ChT, Klumpp H, Elias J, Burns $\mathrm{M}$, et al. Attention training in individuals with generalized social phobia: A randomized controlled trial. J Consult Clin Psychol. 2009;77(5):961-73.

38- Hofmann SG, Sawyer AT, Witt AA, Oh D. The effect of mindfulness-based therapy on anxiety and depression: Ameta-analytic review. J Consult Clin Psychol. 2010;78(2):169-83.

39- Khoury B1, Lecomte T, Fortin G, Masse M, Therien P, Bouchard $\mathrm{V}$, et al. Mindfulness-based therapy: Acomprehensive meta-analysis. Clin Psychol Rev. 2013;33(6):763-71.

40- Ost LG. Efficacy of the third wave of behavioral therapies: Asystematic review and meta-analysis. Behav Res Ther. 2008;46(2):296-321.

41- Eisendrath S, Chartier M, McLane M. Adapting mindfulness-based cognitive therapy for treatmentresistant depression: A clinical case study. Cogn Behav Pract. 2011;18(3):362-370.

42- Brown KW, Ryan RM. The benefits of being present: mindfulness and its role in psychological well-being. J Pers Soc Psychol. 2003;84(4):822-48.

43- Smits JA, Minhajuddin A, Jarrett RB. Cognitive therapy for depressed adults with comorbid social phobia. J Affect Disord. 2010;114(1-3):271-8.

44- Hazlett-Stevens H. Mindfulness-based stress reduction for comorbid anxiety and depression: Case report and clinial considerations. J Nerv Ment Dis. 2012;200(11):999-1003.

45- Ariana-Kia E, Moradi AR, Hatami M. The effectivness of combined of brief behavioral activation therapy and mindfulness-based cognitive therapy in patients with major depressive disorder. J Clin Psychol. 2014;6(1):1527. [Persian]

46- Dimidjian S, Beck A, Felder JN, Boggs JM, Gallop R, Segal Z. Web-based Mindfulness-based Cognitive Therapy for reducing residual depressive symptoms: An open trial and quasi-experimental comparison to propensity score matched controls. Behav Res Ther. 2014;63:83-9.

47- Rapee RM, Spence SH. The etiology of social phobia: Empirical evidence and an initial model. Clin Psychol Rev. 2004;24(7):737-67.

48- Akiskal H. Mood disorder. In: Kaplan HI, Sadock BJ. Kaplan \& Sadock's Comprehensive textbook of psychiatry. Baltimore: Lippincott Williams \& Wilkins; 2005. pp. 1284-1431

49- Ballinger B, Yalom I. Group therapy in practice. In: Bongar B, Beutler LE, editors. Comprehensive Textbook of Psychotherapy: Theory and Practice (1 ${ }^{\text {st }}$ Edition). New York: Oxford University Press. 1995.

50- Hashemi Z, Mahmood-Aliloo M, Hashemi-Nosratabad $\mathrm{T}$. The effectiveness of meta cognitive therapy on major depression disorder: A case report. J Clin Psychol. 2011;2(3):85-97. [Persian]

51- Sharifi V, Asadi SM, Mohammadi MR, Amini H, Kavian H, Semnani Y, et al. Reliability and feasibility of the persian version of the structured diagnostic interview for DSM-IV. Adv Cogn Sci. 2004;6(1\&2):10-22. [Persian] 52- Ostovar S, Razavyeh A. Examine the psychometric properties of the social snxiety scale for adolescents
18- Rodebaugh TL, Holaway RM, Heimberg RG. The treatment of social anxiety disorder. Clin Psychol Rev. 2004;24(7):883-908

19- Ottenbreit ND, Dobson KS. Avoidance and depression: The construction of the cognitive-behavioral avoidance scale. Behav Res Ther. 2004;42(3):293-313.

20- Kashdan TB, Breen WE, Afram A, Terhar D. Experiential avoidance in idiographic, autobiographical memories: Construct validity and links to social anxiety, depressive, and anger symptoms. J Anxiety Disord. 2010;24(5):528-34.

21- Backs-Dermott BJ, Dobson KS, Jones SL. An evaluation of an integrated model of relapse in depression. J Affect Disord. 2010;124(1-2):60-7.

22- Wong QJ, Moulds ML. Impact of rumination versus distraction on anxiety and maladaptive self-beliefs in socially anxiety individuals. Behav Res Ther. 2009;47(10):861-7.

23- Ciesla JA, Felton JW, Roberts JE. Testing the cognitive catalyst model of depression: does rumination amplify the impact of cognitive diatheses in response to stress?. Cogn Emot. 2011;25(8):1349-57.

24- Dickson K.S, Ciesla JA, Reilly LC. Rumination, worry, cognitive avoidance, and behavioral avoidance: Examination of temporal effects. Behav Ther. 2012;43(3):629-40.

25- Kao CM, Dritschel BH, Astell A. The effects of rumination and distraction on over- general autobiographical memory retrieval during social problem solving. Br J Clin Psychol. 2006;45(Pt 2):267-72. 26- McLaughlin KA, Nolen-Hoeksema S. Rumination as a transdiagnostic factor in depression and anxiety. Behav Res Ther. 2011;49(3):186-93.

27- Hong RY, Worry and rumination:differentional associations with anxiety and depressive symptoms and coping behavior. Behav Res Ther. 2007;45(2):277-90.

28- Sarin S, Abela J, Auerbach R. The response styles theory of depression: A test of specificity and causal mediation. J Cogn Emot. 2005;19(5):751-61.

29- Hofman SG. Cognitive factors that maintain social anxiety disorder: A comprehensive model and its treatment implication. Cogn Behav Ther. 2007;36(4):195-209.

30- Smith JM, Alloy LB. A roadmap to rumination: A review of the definition, assessment, and conceptualization of this multifaceted construct. Clin Psycho Rev. 2009;29(2):116-22.

31- Nolen-Hoeksema $S$. The role of rumination in depressive disorder and mixed anxiety/ depressive symptoms. J Abnorm Psychol. 2000;109(3):504-11.

32- Kashdan TB, Roberts JE. Comorbid social anxiety disorder in clients with depressive disorders: Predicting changes in depressive symptoms, therapeutic relationships, and focus of attention in group treatment. Behav Res Ther. 2011;49(12):875-84.

33- Albert U, Rosso G, Maina G, Bogetto F. Impact of anxiety disorder comorbidity on quality of life ineuthymic bipolar disorder patiens: differences between bipolar I and II subtypes. J Affect Disord. 2008;105(1-3):297-303.

34- Schoevers RA, Deeg DJ, van Tilburg W, Beekman AT. Depression and generali anxiety disorder. Co-occurrence and longituinal patiens inelderly patiens. Am J Geriatr Psychiatry. 2005;13(1):31-9.

35- Hamilton K, Wershler JL, Macrodimitris SD, Ching LE, Mothersill KJ. Exploring the effectiveness of a mixeddiagnosis group cognitive behavioral therapy 
55- Teasdale JD, Segal ZV, Williams JM, Ridgeway VA Soulsby JM, Lau MA. Prevention of relapse/recurrence in major depression by mindfulness-based cognitive therapy. J Consult Clin Psychol. 2000;68(4):615-23.

56- Geschwind N, Peeters F, Huibers M, Van Os J, Wichers M. Efficacy of mindfulness-based cognitive therapy in relation to prior history of depression: Randomised controlled trial. Br J Psychiatry. 2012;201(4):320-5.

57- Bohlmeijer E, Prenger R, Taal E, Cuijpers P. The effects of mindfulness-based stress reduction therapy on mental health of adults with achronic medical disease: A meta-analysis. J Psychosom Res. 2010;68(6):539-44.
اع ع عزتاله قدميور و همكاران

(SAS-A) for use in Iran. J Psychol Model Method. 2013;3(12):69-78. [Persian]

53- Moulds ML, Kandris E, Starr S, Wong ACM. The relationship between rumination, avoidance and depression in a non-clinical sample. Behav Res Ther. 2007;45(2):251-61.

54- Ataie Sh, Fata L, Ahmadi Abhari A. Rumination and cognitive behavioral avoidance in depressive and social anxiety disorders: Comparison between dimensional and categorical approaches. Iran J Psychiatry Clin Psychol. 2014;19(4):283-92. [Persian] 